\title{
PFGE and antibiotic susceptibility phenotype analysis of Pseudomonas aeruginosa strain chronically infecting Cystic Fibrosis patients
}

\author{
Giovanna Pulcrano', Fabio Rossano', Antonietta Lambiase', Mariassunta Del Pezzo', \\ Emanuela Roscetto',Valeria Raia', Maria Rosaria Catania' \\ 'Dipartimento di Biologia e Patologia Cellulare e Molecolare “Luigi Califano”, Facoltà di Medicina e Chirurgia, Università \\ di Napoli "Federico II"; \\ ${ }^{2}$ Centro di Riferimento Regionale per la Fibrosi Cistica, Dipartimento di Pediatria, Facoltà di Medicina e Chirurgia, \\ Università di Napoli "Federico II".
}

Key words: Cystic Fibrosis, antimicrobial susceptibility, PFGE, chronic colonization.

Analisi delle caratteristiche fenotipiche e genotipiche di ceppi sequenziali di Pseudomonas aeruginosa isolati da pazienti con fibrosi cistica

\section{SUMMARY}

Pseudomonas aeruginosa is the leading cause of chronic lung infection and following pulmonary worsening of cystic fibrosis patients. To verify whether bacterial modifications regarding motility, mucoidy, and serum susceptibility proceeded from an adaptation to chronic infection or a replacement with a new strain, sequential $P$. aeruginosa isolates of known phenotype collected from 5 cystic fibrosis patients were typed by pulsed-field gel electophoresis (PFGE). Antimicrobial susceptibility testing of all isolates was performed by the disc diffusion method. PFGE typing demonstrated that strains dissimilar in colony morphotype and of different antibiotic susceptibility patterns could be of the same genotype. Some patients were colonized with a rather constant $P$. aeruginosa flora, with strains of different phenotypes but of one genotype. Instead, some patients may be colonized by more than one genotype. Secretion of mucoid exopolysaccharide and acquisition of a new antibiotic susceptibility phenotype in these strain appear to evolve during chronic colonization in cystic fibrosis patients from specific adaptation to infection rather than from acquisition of new bacterial strains.

\section{INTRODUZIONE}

Pseudomonas aeruginosa (PA) è un microrganismo Gram-negativo ambientale, conosciuto da tempo come patogeno responsabile di infezioni opportunistiche in pazienti ospedalizzati e in soggetti affetti da fibrosi cistica (FC). In questi pazienti, il microrganismo, acquisito casualmente da sorgenti ambientali, presenta inizialmente una chemiosensibilità variabile e raramente un fenotipo mucoide $(2,6,9)$. Una volta che il microrganismo ha colonizzato in maniera cronica l'epitelio respiratorio diventa impossibile eradicarlo tramite terapia antibiotica $(3,7,10)$. Dopo diversi trattamenti terapeutici appaiono varianti multi-resistenti e con fenotipo mucoide. La morfologia mucoide di questi ceppi è strettamente correlata alla capacità di formare biofilm e quindi ad una aumentata poliantibiotico-resistenza (8). Tra i fattori che contribuiscono a rendere un ceppo di PA più o meno virulento, il gene pilA, coinvolto nella biosintesi del pilo e quindi nei meccanismi di quorum-sensing e nella formazione del biofilm stesso, ha un ruolo fondamentale $(1,5)$. È riportato in letteratura che ceppi di PA isolati da pazienti affetti da FC presentino geni pilA classificabili in pochi gruppi filogenetici e nettamente differenti da quelli dei ceppi ambientali (4).

La tipizzazione dei ceppi di PA nei pazienti affetti da FC è fondamentale al fine di monitorare la possibilità di cross-infezioni tra pazienti ricoverati negli stessi centri. Tale tipizzazione non si avvale delle classiche metodologie fenotipiche ma di analisi genotipiche più discriminanti (16). Diversi studi hanno analizzato la diversità e l'evoluzione

\section{Corresponding author: Giovanna Pulcrano}

Dipartimento di Biologia e Patologia Cellulare e Molecolare "L. Califano"

Facoltà di Medicina e Chirurgia, Università di Napoli "Federico II" - Via Pansini 5 - 80I 3 I-Napoli

Tel.: 08 I 7464343 - Fax:08I 7462530 - e-mail: giovannapulcrano@libero.it 
dei ceppi che colonizzano cronicamente le vie respiratorie dei pazienti affetti da FC mediante analisi dei polimorfismi di lunghezza dei frammenti di restrizione (RFLP) in geni ipervariabili come il gene pilA o il gene per l'esotossina A oppure tramite analisi del pattern di restrizione per PFGE (13).

Questi studi hanno riportato casi di infezione cronica sostenuta da parte di un singolo ceppo, casi in cui la colonizzazione da parte di un ceppo predominante periodicamente è stata rimpiazzata da ceppi transitori oppure casi di colonizzazione de parte di ceppi multipli.

La correlazione tra acquisizione o perdita di antibiotico-resistenze in ceppi isolati in sequenza da uno stesso paziente e correlazione tra i ceppi hanno evidenziato che nel $20 \%$ dei casi le modifiche del pattern di antibiotico-sensibilità sono associate a sostituzioni del ceppo con un altro, mentre nel $65 \%$ dei casi le modificazioni erano solo temporanee e legate a mutazioni nello stesso ceppo localizzate in geni determinanti delle resistenze antibiotiche, come le beta-lattamasi inducibili. Lo scopo del nostro lavoro è mettere a confronto alcuni ceppi di PA isolati con frequenza trimestrale dagli espettorati di pazienti affetti da FC colonizzati in modo cronico, correlando le variazioni del fenotipo e del profilo di antibiotico-sensibilità con il profilo di macrorestrizione.

\section{MATERIALI E METODI \\ Pazienti e isolati}

Sono stati inclusi nello studio 24 ceppi isolati nell'arco di 3 anni da 5 pazienti affetti da FC colonizzati in modo cronico da PA. I pazienti, monitorati a intervalli regolari di 3 mesi, presentavano infatti almeno un isolamento di PA. I ceppi sono stati isolati dagli espettorati di pazienti monitorati nel Centro di Riferimento Regionale per la Fibrosi Cistica presso il Dipartimento di Pediatria del Policlinico "Federico II". Tutti i ceppi sono stati identificati mediante il sistema automatizzato BD Phoenix.

\section{Test di antibiotico-sensibilità}

I ceppi in esame sono stati sottoposti a test di antibiotico-sensibilità su Mueller-Hinton Agar secondo il metodo Kirby-Bauer. Sono stati utilizzati i seguenti antibiotici: amikacina, ampicillina, aztreonam, cefepime, ceftazidime, ciprofloxacina, cloramfenicolo, gentamicina, meropenem, levofloxacina, piperacillina, piperacillina/tazobactam, tetraciclina.

\section{PFGE}

I ceppi in esame sono cresciuti fino ad una O.D. $10^{7} \mathrm{CFU} / \mathrm{ml}$, quindi inclusi in blocchetti di agarosio al $1.6 \%$ per preparare i plug. I plug solidificati sono sottoposti a lisi con lisozima $(2 \mathrm{mg} / \mathrm{ml})$ e proteinasi K (1mg/ml). Successivamente, dopo 56 lavaggi in buffer TE (Tris $10 \mathrm{mM} \mathrm{pH} 7.5$, EDTA $1 \mathrm{mM} \mathrm{pH} \mathrm{8)} \mathrm{e} \mathrm{digestione} \mathrm{con} \mathrm{enzima} \mathrm{di} \mathrm{restrizio-}$ ne SpeI, è stata eseguita un'elettroforesi con un initial switch time di $5 \mathrm{sec}$ ed un final switch time di $35 \mathrm{sec}$ per 20 ore (14). I prodotti della restrizione sono stati analizzati secondo i criteri di Tenover per cui, secondo il numero di bande riscontrate, è possibile definire le condizioni di correlazione tra i ceppi (15).

\section{RISULTATI}

L'attenzione è stata focalizzata su cinque pazienti del Centro di Fibrosi Cistica del Policlinico "Federico II" colonizzati da ceppi di PA non correlati tra loro e caratterizzati da diversi geni pilA (11) e profili di antibiotico-sensibilità piuttosto differenti, come riportato in tabella 1 . I ceppi in esame sono stati indicati con le sigle A1-3, G1-4, MR1-6, MH1-6 e T1-5, indicando con la lettera il ceppo isolato dallo stesso paziente e con il numero progressivo gli isolati in successione temporale.

La prima osservazione è stata fatta sul fenotipo: tutti i ceppi isolati dai pazienti A, G e T si presentano mucoidi, mentre quelli isolati dagli altri due pazienti mostrano questo fenotipo dopo alcuni mesi dal primo isolamento. Per quanto riguarda il profilo di antibiotico-sensibilità, solo i ceppi del paziente $G$ non presentano una variabilità nel corso del tempo, mentre per i ceppi dei pazienti MR e T si osservano poche variazioni relative solo agli antibiotici in particolare per gli antibiotici gentamicina, piperacillina e piperacillina/tazobactam. L'antibiotico-sensibilità dei ceppi degli altri due pazienti è molto variabile nel corso del tempo. L'analisi della macrorestrizione ha dimostrato che i pazienti $\mathrm{A}, \mathrm{G}$ e $\mathrm{T}$ sono cronicamente colonizzati con isolati appartenenti allo stesso "lineage" per prolungati periodi di tempo, mentre, nel caso del paziente MR, il ceppo colonizzante, identico nel pattern di restrizione nei primi tre isolati, presenta una banda aggiuntiva nel quarto. Il successivo isolato, MR5, non è da ritenersi correlato ai precedenti, mentre l'isolato MR6 risulta identico al quarto (figura I). Il paziente $\mathrm{MH}$, allo stesso modo, risulta colonizzato da un ceppo predominante (MH1-2, MH4-6) e un ceppo transitorio (MH3).

\section{CONCLUSIONI}

Ceppi di PA isolati da pazienti affetti da FC e colonizzati in maniera cronica sono caratterizzati da aspetti fenotipici differenti da altri ceppi isolati da altri pazienti o dall'ambiente esterno. Indagini epidemiologiche su questi ceppi possono aiutare a meglio capire il loro ruolo nella progressione delle infezioni respiratorie nella malattia. 
Tabella I. Sensibilità agli antibiotici e caratteristiche fenotipiche dei ceppi analizzati

\begin{tabular}{|c|c|c|c|c|c|c|c|c|c|c|c|c|c|c|c|}
\hline & notipo & Pilina & AMK & AMP & ATM & FEP & CAZ & CIP & CHL & GEN & MEM & LVX & PIP & TZP & TET \\
\hline$\overline{A I}$ & $M$ & PAK & $R$ & $\mathrm{R}$ & $S$ & $S$ & $R$ & $S$ & $\mathrm{R}$ & $R$ & $R$ & $S$ & $\mathrm{R}$ & 1 & $R$ \\
\hline A2 & $M$ & & $S$ & $\mathrm{R}$ & $R$ & $S$ & $\mathrm{R}$ & $S$ & $\mathrm{R}$ & $\mathrm{R}$ & $R$ & $S$ & $\mathrm{R}$ & $S$ & $\mathrm{R}$ \\
\hline A3 & $M$ & & $S$ & $R$ & $S$ & $S$ & $R$ & $S$ & $\mathrm{R}$ & $R$ & $R$ & $S$ & $R$ & $R$ & $R$ \\
\hline$\overline{\mathbf{G I}}$ & $M$ & $\mathrm{~Pa} \mid 4$ & $S$ & $\mathrm{R}$ & $S$ & $S$ & $S$ & $S$ & $R$ & $S$ & $S$ & $S$ & $S$ & $S$ & $R$ \\
\hline G2 & $M$ & & $S$ & $\mathrm{R}$ & $S$ & $S$ & $S$ & $S$ & $\mathrm{R}$ & $S$ & $S$ & $S$ & $S$ & $S$ & $\mathrm{R}$ \\
\hline$\overline{\mathbf{G 3}}$ & $M$ & & $S$ & $R$ & $S$ & $S$ & $S$ & $S$ & $R$ & $S$ & $S$ & $S$ & $S$ & $S$ & $R$ \\
\hline$\overline{\text { G4 }}$ & $M$ & & $S$ & $R$ & $S$ & $S$ & $S$ & $S$ & $R$ & $S$ & $S$ & $S$ & $S$ & $S$ & $R$ \\
\hline MHI & $\mathrm{R}$ & PAK & $R$ & $R$ & $S$ & $R$ & $S$ & $S$ & $R$ & $R$ & $\mathrm{R}$ & $S$ & $R$ & $S$ & $R$ \\
\hline $\mathrm{MH2}$ & $\mathrm{R}$ & & $S$ & $R$ & $S$ & $R$ & $S$ & $S$ & $R$ & $R$ & $S$ & $S$ & $S$ & $S$ & $R$ \\
\hline$\overline{\mathrm{MH} 3}$ & $M$ & & $S$ & $R$ & $S$ & $S$ & $S$ & $S$ & $R$ & $S$ & $S$ & $S$ & $S$ & $S$ & $R$ \\
\hline MH4 & $M$ & & $S$ & $R$ & $S$ & $R$ & $S$ & $S$ & $\mathrm{R}$ & $R$ & $S$ & $S$ & $S$ & $S$ & $\mathrm{R}$ \\
\hline MH5 & $M$ & & $S$ & $R$ & $R$ & $S$ & $S$ & $S$ & $R$ & $S$ & $S$ & $S$ & $R$ & $S$ & $R$ \\
\hline MH6 & $M$ & & $S$ & $R$ & $S$ & $S$ & $S$ & $S$ & $R$ & $R$ & $S$ & $S$ & $S$ & $S$ & $R$ \\
\hline MRI & $\mathrm{R}$ & PAK & $R$ & $R$ & $R$ & $R$ & $R$ & $R$ & $R$ & $S$ & $R$ & $R$ & $R$ & $S$ & $R$ \\
\hline MR2 & $\mathrm{R}$ & & $R$ & $R$ & $R$ & $R$ & $R$ & $R$ & $R$ & $R$ & $R$ & $R$ & $R$ & $S$ & $R$ \\
\hline MR3 & $\mathrm{R}$ & & $R$ & $R$ & $R$ & $R$ & $R$ & $R$ & $R$ & $R$ & $R$ & $R$ & $\mathrm{R}$ & $S$ & $R$ \\
\hline MR4 & $\mathrm{R}$ & & $R$ & $\mathrm{R}$ & $R$ & $R$ & $R$ & $R$ & $R$ & $R$ & $\mathrm{R}$ & $R$ & $R$ & $R$ & $R$ \\
\hline MR5 & $M$ & & $R$ & $R$ & $R$ & $R$ & $R$ & $R$ & $R$ & $R$ & $R$ & $R$ & $R$ & $R$ & $R$ \\
\hline MR6 & $M$ & & $R$ & $R$ & $R$ & $R$ & $R$ & $R$ & $\mathrm{R}$ & $R$ & $R$ & $R$ & $R$ & $R$ & $R$ \\
\hline TI & $M$ & $\mathrm{PaI03}$ & $S$ & $\mathrm{R}$ & $S$ & $\mathrm{R}$ & $S$ & $S$ & $R$ & $\mathrm{R}$ & $S$ & $S$ & $R$ & $R$ & $R$ \\
\hline$\overline{\text { T2 }}$ & $M$ & & $S$ & $R$ & $S$ & $R$ & $S$ & $S$ & $R$ & $R$ & $S$ & $S$ & $R$ & $R$ & $R$ \\
\hline$\overline{\text { T3 }}$ & $M$ & & $S$ & $R$ & $S$ & $R$ & $S$ & $S$ & $\mathrm{R}$ & $R$ & $S$ & $S$ & $R$ & $R$ & $R$ \\
\hline T4 & $M$ & & $S$ & $\mathrm{R}$ & $S$ & $R$ & $S$ & $S$ & $\mathrm{R}$ & $\mathrm{R}$ & $S$ & $S$ & $S$ & $S$ & $R$ \\
\hline T5 & $M$ & & $S$ & $\mathrm{R}$ & $S$ & $R$ & $S$ & $S$ & $R$ & $\mathrm{R}$ & $S$ & $S$ & $S$ & $S$ & $R$ \\
\hline
\end{tabular}

AMK=Amikacina;AMP=Ampicillina;ATM=Aztreonam; FEP= Cefepime; CAZ=Ceftazidime; CIP=Ciprofloxacina;

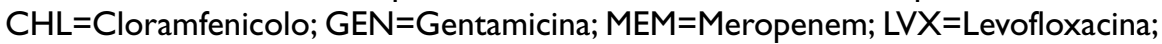

$\mathrm{PIP}=$ Piperacillina; $\mathrm{TZP}=$ Piperacillina/tazobactam TET=Tetraciclina.

$\mathrm{M}$ : fenotipo mucoide. R: fenotipo rugoso.

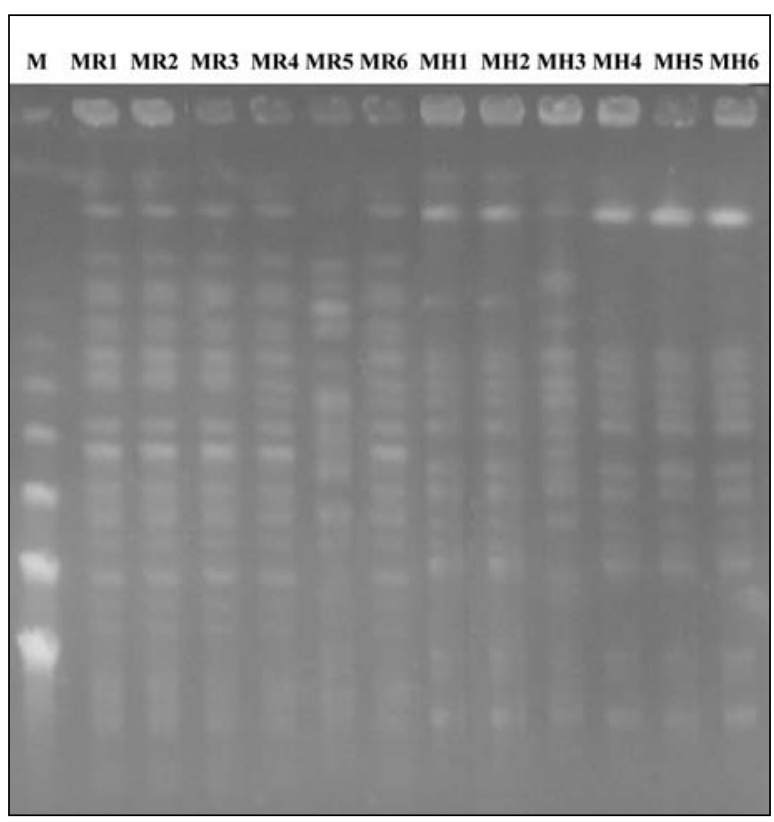

Figura I. Profilo PFGE di ceppi di Pseudomonas aeruginosa isolati dai pazienti MR e MH. M: marcatore di peso molecolare.

L'incremento notevole di microrganismi poli-antibiotico resistenti, con fenotipo mucoide è un importante aspetto da monitorare e la caratterizzazione molecolare è una metodologia in grado di dare le informazioni riguardanti lo "stato" del ceppo e la sua potenzialità nel diffondere nella popolazione dei pazienti FC.

La tipizzazione tramite metodi fenotipici convenzionali risulta inattendibile; invece analisi genotipiche risultano più semplici e riproducibili nonché indispensabili per differenziare ceppi non correlati e studiare l'epidemiologia dell'infezione nei pazienti. Spesso alterazioni nell'espressione del mucopolisaccaride, nella motilità batterica o nel profilo di antibiotico-sensibilità non corrispondono a variazioni del profilo di restrizione che rimane stabile in isolati raccolti in sequenza, suggerendo che queste alterazioni fenotipiche possono essere il risultato di una risposta adattativa dell'isolato colonizzante.

Dal nostro studio, in accordo con dati di letteratura $(2,9)$, risulta evidente che alcuni pazienti sono colonizzati da un unico ceppo nel corso di lunghi periodi di tempo; al contrario altri pazienti presentano sovrainfezioni da parte di ceppi transitori, pur conservando un unico ceppo predominante. Non sono stati qui analizzati casi di pazienti colonizzati da più ceppi contemporaneamente, pur essendo stati isolati nel corso degli anni ceppi multipli dallo stesso paziente. In questo studio abbiamo anche analizzato la correlazione tra la variazione nel profilo dell'antibiotico-sensibilità 
in isolati prelevati in modo sequenziale e il livello di correlazione genetica, mettendo in evidenza che spesso questa variabilità non corrisponde ad un'uguale variabilità nel pattern di restrizione. In parallelo, evidenti mutazioni del pattern di restrizione non corrispondono a modifiche dell'antibiotico-sensibilità (paziente MR). Questi dati supportano la conclusione avanzata già da Römling (12) e collaboratori che, in pazienti affetti da FC, isolati geneticamente diversi di PA sviluppano un fenotipo comune nel corso di infezione cronica.

\section{BIBLIOGRAFIA}

1. Farinha MA, Ronald SL, Kropinski AM, Paranchych W. Localization of the virulence-associated genes pilA, pilR, rpoN, fliA, fliC, ent, and fbp on the physical map of Pseudomonas aeruginosa PAO1 by pulsedfield electrophoresis. Infect Immun 1993; 61: 1571-5.

2. Gómez MI, Prince A. Opportunistic infections in lung disease: Pseudomonas infections in cystic fibrosis. Curr Opin Pharmacol 2007; 7: 244-51.

3. Hoiby N, Frederiksen B, Pressler T. Eradication of early Pseudomonas aeruginosa infection. J Cyst Fibros 2005; 2: 49-54.

4. Kus JV, Tullis E, Cvitkovitch DG, Burrows LL. Significant differences in type IV pilin allele distribution among Pseudomonas aeruginosa isolates from cystic fibrosis (CF) versus non-CF patients. Microbiology 2004; 150: 1315-26.

5. Le Berre R, Nguyen S, Nowak E, et al. Quorum-sensing activity and related virulence factor expression in clinically pathogenic isolates of Pseudomonas aeruginosa. Clin Microbiol Infect 2008; Jan.

6. Lyczak JB, Cannon CL, Pier GB. Lung infections associated with cystic fibrosis. Clin Microbiol Rev 2002; 15: 194-222.
7. Mahenthiralingam E, Campbell ME, Speert DP. Nonmotility and phagocytic resistance of Pseudomonas aeruginosa isolates from chronically colonized patients with cystic fibrosis. Infect Immun 1994; 62: 596-605.

8. Mathee K, Ciofu O, Sternberg C, et al. Mucoid conversion of Pseudomonas aeruginosa by hydrogen peroxide: a mechanism for virulence activation in the cystic fibrosis lung. Microbiology 1999; $145: 1349-57$.

9. Murray TS, Egan M, Kazmierczak BI. Pseudomonas aeruginosa chronic colonization in cystic fibrosis patients. Curr Opin Pediatr 2007; 19: 83-8.

10. Pier GB, Meluleni G, Neuger E. A murine model of chronic mucosal colonization by Pseudomonas aeruginosa. Infect. Immun 1992; 60: 4768-76.

11. Pulcrano G, Lambiase A, Del Pezzo M, Raia V, Rossano F. Characterization of different Pseudomonas aeruginosa strain in Cystic Fibrosis patients. Microbiologia Medica 2007; 22: 115-8.

12. Römling U, Fiedler B, Bosshammer $J$, et al. Epidemiology of chronic Pseudomonas aeruginosa infections in cystic fibrosis. J Infect Dis. 1994; 170: 1616-21.

13. Speert DP, Campbell ME, Farmer SW, Volpel K, Joffe AM, Paranchych W. Use of a pilin gene probe to study molecular epidemiology of Pseudomonas aeruginosa. J Clin Microbiol 1989; 27: 2589-93.

14. Tenover F, Arbeit R, Goering R, et al. Interpreting chromosomal DNA restriction patterns produced by pulsed-field gel electrophoresis: criteria for bacterial strain typing. J Clin Microbiol 1995; 33: 2233-9.

15. Tenover F, Arbeit R, Goering RV. How to select and interpret molecular strain typing methods for epidemiological studies of bacterial infections: a review for healthcare epidemiologists. Infect. Control Hosp. Epidemiol 1997; 18: 426-39.

16. The International Pseudomonas aeruginosa Typing Study Group. A multicenter comparison of methods for typing strains of Pseudomonas aeruginosa predominantly from patients with cystic fibrosis. J Infect Dis 1994; 169: 134-42. 